\title{
Biomedical Signal Processing, Artificial Neural Network: A Review
}

\author{
Chhatrapal Singh* and Jaspinder Singh \\ Department of Electronics and Communication systems, Lovely Professional University, Punjab, \\ India; singh.cp002@gmail.com, 2jaspinder.19601@|pu.co.in
}

\begin{abstract}
Feature extraction of biomedical signals plays a significant role in diagnosing of diseases or problems. This paper shows some basic features and processing techniques for biomedical signals and also contains the brief description of neural networks. The biomedical signals are related to the body organs which describe electrically working of associated organs. Examples of biomedical signals are EEG, ECG, EMG. For classifying the biomedical signals, we use ANNs because classification is the most growing research and application area of neural network. Neural networks are used in lot of areas of industry like finance, engineering, biology. Here describes the ANN and its importance. For solving the nonlinear classification problem, used BPNN which is multi- layer feed forward neural network.
\end{abstract}

Keywords: Artificial Neural Networks (Anns), Biomedical Signal, BPPN Algorithm, EEG, ECG, Wavelet Transforms

\section{Introduction}

In this paper, two different topics are explained. First section of the paper contains the brief study of biomedical signals and their type. And also provide small over view of the wavelet transform. Second section of this paper explains the concept of ANNs and working of multi-layer neural networks.

\section{Biomedical Signals}

Biomedical science is a branch of science where we deal with the body organs. For processing the information we required data in the form of image, text, audio and video. But in biomedical we collect the information in the form of image and signal of electrical change. Depending upon the body organs, application or signal characteristics biomedical signals are classified like EOG, ENG, EGG, ECG, EMG, EEG, CP etc. But ECG, EMG and EEG signals are mostly used for research areas of biomedical field.

\subsection{Electrocardiogram (ECG) Signal}

This signal is related to the cardiac system of body which are generated by the depolarization and repolarization of the atria and ventricles. ECG signal is a combination waves. They waves are P-QRS-T. In ECG signal we have focus on the amplitudes and intervals of pulse or waves.

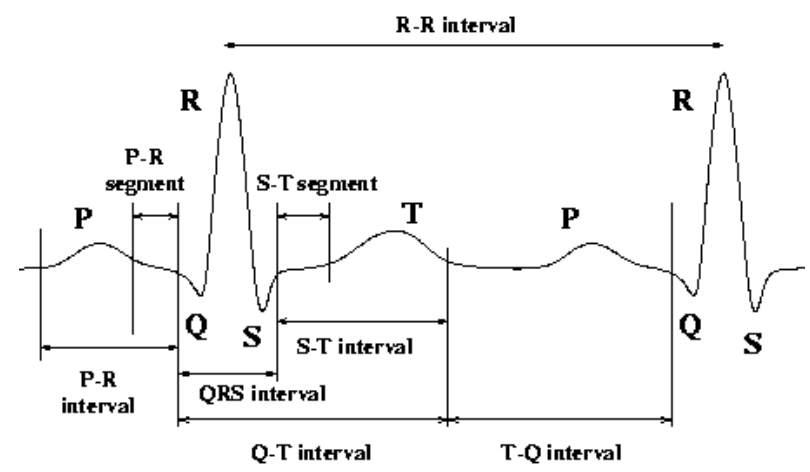

Figure 1. ECG signal of human cardiac system.

For extraction of feature from the ECG signal we have many techniques now days in both domains, time domain as well as frequency domain - .- Magnitude of ECG signal is just potential difference between heart layers. It taken by the 12 different leads (angles) and recorded over nearly 10 second time duration. For recording of ECG signal, 12 leads are used a electrodes which are placed on the patient body at different places, 


\subsection{Electroencephalography (EEG) Signal}

It is an electro physiological monitoring process to record electrical activity of the brain. The brain has billions of neurons which are electrical charged. So we get the different frequency or waves at different condition of brain. It is measures by the placing electrodes along with the Scalp. EEG measured voltage fluctuation between neuron of brain.

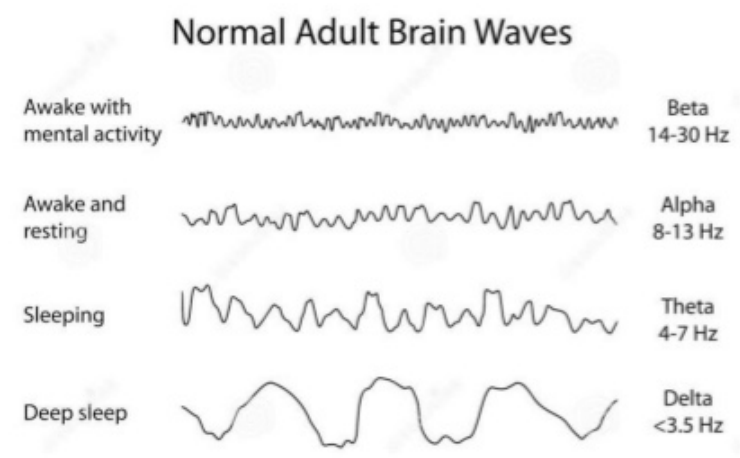

Figure 2. EEG signal of human brain.

EEG signal are associated with different actions in the brain. EEG signal has a range of frequency 0 to $100 \mathrm{~Hz}$; there are six type of frequency patterns

a. Delta up to $4 \mathrm{~Hz}$.

b. Theta range is $4 \mathrm{~Hz}-7 \mathrm{~Hz}$

c. Alpha range is $7 \mathrm{~Hz}-14 \mathrm{~Hz}$

d. Beta range is $15 \mathrm{~Hz}-30 \mathrm{~Hz}$.

e. Gamma range is $30 \mathrm{~Hz}-100 \mathrm{~Hz}$.

f. Mu range is $8 \mathrm{~Hz}-13 \mathrm{~Hz}$.

For recording of EEG signal it required long time connection to the subject and dozens electrodes around the head.

\subsection{Wavelet Transforms}

Wavelet transforms are based on small waves, called wavelets. Wavelets are function that satisfies certain requirements. They divided the data into different frequency components and, study each component with a resolution matched to its scale. Wavelet algorithms process data at different scales or resolutions. In wavelet transforms data divided into different frequency component like low frequency and high frequency.

\section{Artificial Neural Networks (ANNs)}

ANN concept is basically taking from biology. Artificial neural networks are used to approximating the relationship between input and output signal of the network. It provides a mathematical and computational model for processing of information.

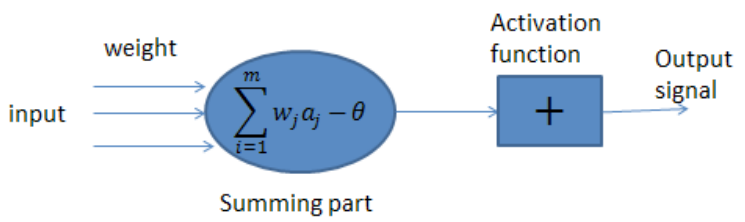

Figure 3. Block diagram of ANN

It has device with many inputs and one output. Similar to biological neuron ANN also receives inputs from the other elements or other ANN and after the inputs are weighted and the result is then transformed by a transfer function into output [6].

\subsection{ANN Characteristics}

Computers are good in calculations but they follow particular algorithms which are programmed but ANNs improve their own rules and making better decision. The characteristic of artificial neural networks are very similar to an intelligent system and other artificial intelligence based applications?.

There are five characteristics of ANNs.

- Networks Type - The network structure of ANN kept as simple and easy as. Basically two type of structures Feedback Network and Feed Forward Networks. Signal moving in one direction in Feed forward Network and signal can travel in both ways in Feedback Network

- Processing - Neural networks can perform massively parallel operations. Parallel processing in human body is very complex but using some parallel processing techniques, we can capable to implement it in ANNs

- Storage- Neural networks store information in the 
strengths of the interconnections. Information is stored in the form of weight matrix

- Fault Tolerance Ability - ANN is a complex network so it should kept fault tolerance ability. So if any connection is missing then output should not affect and system can work properly. If many connections are fails then system also fails at the same time日.

- Learning Ability - Number of algorithms are present to train the networks. In ANNs, three learning methods are used they are Supervised learning, Unsupervised learning and Reinforcement learning

\subsection{Multi-Layer Feed Forward Neural Network}

Multi-layer neural is taken for solving the nonlinear problems. It contains three layers to processing the data input to output layer. One in input layer where input data is providing to the network. Second layer is hidden layer; in this network calculated the weights of the system and error correction process take place. Third layer is output layer which provide the result or problem or networks,

Back Propagation Neural network (BPN) is a multilayer feed forward neural network. For training of BPN is done in three phase

- Feed forward phage.

- Calculation and back propagation of error phage.

- Updation of weights and Biasing.

\section{Literture Review}

This section of paper discusses various techniques or transformations earlier for extracting the feature from the biomedical signal.

Applied multi-scale principal component analysis for quality controlled de-noising of multichannel Electrocardiogram signals. Collecting wavelet coefficients of all ECG channels at a wavelet scale multivariate data matrices were formed. Principal component analysis was performed on these matrices for signal denoising

Another method to extracting features from the biomedical signal is one- step wavelet. In this processing for ranging and noise removing in ECG signals technique illustrated the application of the DWT to the processing of ECG for ranging and noise suppression in this paper. In scheme allowed to less computational complexity, while its fixed-point modeling showed the expected performance of possible future portable hardware implementations.
The system had been tested using synthetic ECG signals, which allowed to accurately measuring the effect of the proposed processing.

Developed a technique to automatically detect and mark the basic waveforms of ECG signal. Recently developed PCA had been used for this purpose.

We can de-noise the biomedical signal using DWT also in which they considered that the DWT based wavelet de-noising had incorporated using different thresholding techniques to remove noises from the acquired ECG signals like power line interference, baseline wandering, and high frequency noises. Three wavelet functions ("db4", "coif5" and "sym7") and four different thresholding methods were used to de-noise the noise in ECG signals

\section{Future Work}

Artificial neural networks can be trained for the nonlinear problems which are difficult for human being to solve. Many ways are present to extract features from signal. By taking a data set of different biomedical signal, ANNs can be trained which can classify the signal features and give a precise decision about signal features.

\section{Conclusion}

In the field of biomedical, number of ways is developed for recording the signals. And also number of transformations has been proposed for extracting feature from the biomedical signal. Some algorithms are required to train the artificial neural networks. Now a day, many new algorithms are developed to train the ANNs for the nonlinear problem with less complexity.

\section{References}

1. Mahmoodabadi SZ, Ahmadian A, Abolhasani MD. ECG feature extraction using daubechies wavelets. Proceedings of the 5th IASTED International Conference on Visualization, Imaging and Image Processing; 2005. p. 343-8.

2. Martinez JP, Almeida R, Olmos S, Rocha AP, Laguna P. A wavelet-based ECG delineator: Evaluation on standard databases. IEEE Transactions on Biomedical Engineering. 2004; 51(4):570-81.

3. Prasad K, Sahambi JS. Classification of ECG arrhythmias using multi-resolution analysis and neural networks. IEEE Transactions on Biomedical Engineering. 2003; 1:227-31.

4. Li C, Zheng C, Tai C. Detection of ECG characteristic 
points using wavelet transforms. IEEE Transactions on Biomedical Engineering. 1995; 42(1):21-8.

5. Jain AK, Mao J, Mohiuddin KM. Artificial neural networks: A tutorial. Michigan State University; 1996.

6. Li EY. Artificial neural networks and their business applications. Taiwan, 1994.

7. Stergiou C, Dimitrios S. Neural networks and its applications.
8. Available from: http://www.idsia.ch/ juergen/rnn.html

9. Gopal S. Artificial Neural Networks for Spatial Data Analysis. Boston. 1988.

10. Sharma LN, Dandapat S, Mahanta A. Multi-scale principal component analysis for multichannel ECG data reduction. Proceedings of the 10th IEEE International Conference on Information Technology and Applications in Biomedical; 2010. p. 1-4. Doi: 10.11.09/ITAB.2010.5687778 